\title{
Coadjuvantes no fortalecimento do sistema imunológico para o enfrentamento ao COVID-19
}

\author{
Helpers in the strengthening of the immunological system to face COVID-19 \\ Ayudantes en el fortalecimiento del sistema inmunológico para enfrentar el COVID-19
}

Recebido: 23/09/2021 | Revisado: 30/09/2021 | Aceito: 04/10/2021 | Publicado: 06/10/2021

Eduarda Moreira Cardoso

ORCID: https://orcid.org/0000-0002-4571-2664

Faculdade Integrada Carajás, Brasil

E-mail: eduardamorcardoso@gmail.com

Gislene Pinto da Silva

ORCID: https://orcid.org/0000-0002-3956-6365

Faculdade Integrada Carajás, Brasil

E-mail: giza.luiza@gmail.com

Jânio Sousa Santos

ORCID: https://orcid.org/0000-0003-2180-1109

Faculdade Integrada Carajás, Brasil

E-mail: santosjs.food@gmail.com

\begin{abstract}
Resumo
O objetivo do presente artigo foi realizar um estudo de nutrientes e fitoterápicos que podem ser utilizados como coadjuvante no auxílio do fortalecimento do sistema imunológico. Portanto trata-se de uma análise bibliográfica descritiva explicativa que incluir revisão bibliográfica e que contém um recorte temporal de 2013 a 2021, as informações obtidas foram retiradas de bases de dados de documentos científicos, selecionando-se documentos descritos nos idiomas inglês e português, que relatasse sobre reações com SARS-CoV-2 a interação de uma alimentação saudável no cotidiano de fato é muito importante além dos outros métodos de cuidado. As plantas medicinais podem confortar e combater várias doenças infecciosas. $\mathrm{O}$ organismo quando se tem uma infecção e bastante vulnerável visto que os microrganismos prejudicam ocasionando deficiências nas células, mesmo que o organismo possuir células que os destrói, contudo precisa-se de nutrientes que transforma o sistema imunológico preferencialmente apto, preservando o equilíbrio para o funcionamento da proteção o sistema imunológico atuam na proteção do organismo diariamente aos ataques contra infecções, quando vírus como o SARS-CoV-2, dominam o organismo, as células entram em ação para combater o vírus e possivelmente obter uma cura ou melhoria do indivíduo. Portanto com base nas literaturas revisadas, concluiu-se que os coadjuvantes como alimentação saudável e fitoterápicos tem disposição de contribuir para reposição de nutrientes capaz de reforçar a imunidade do indivíduo.

Palavras-chave: Coronavírus; Alimento funcional; Suplementos nutricionais; Plantas medicinais; Imunidade.
\end{abstract}

\begin{abstract}
The aim of this article was to carry out a study of nutrients and herbal medicines that can be used as an adjunct to help strengthen the immune system. Therefore, this is an explanatory descriptive bibliographic analysis that includes a bibliographic review and contains a time frame from 2013 to 2021. The information obtained was taken from databases of scientific documents, selecting documents described in English and Portuguese, which reporting on reactions with SARS-CoV-2 the interaction of healthy eating in daily life is indeed very important in addition to other care methods. Medicinal plants can comfort and fight various infectious diseases. The organism, when you have an infection, is quite vulnerable since microorganisms harm causing cell deficiencies, even if the organism has cells that destroy them, however it needs nutrients that make the immune system preferentially fit, preserving the balance for functioning From protection the immune system acts to protect the body daily from attacks against infections, when viruses such as SARS-CoV-2 dominate the body, the cells take action to fight the virus and possibly obtain a cure or improvement for the individual. Therefore, based on the reviewed literature, it was concluded that adjuvants such as healthy eating and herbal medicines are willing to contribute to nutrient replacement capable of strengthening the individual's immunity.
\end{abstract}

Keywords: Coronavirus; Functional food; Dietary supplements; Medicinal plants; Immunity.

\section{Resumen}

El objetivo de este artículo fue realizar un estudio de nutrientes y medicinas a base de hierbas que se pueden utilizar como coadyuvantes para ayudar a fortalecer el sistema inmunológico. Por tanto, se trata de un análisis bibliográfico descriptivo explicativo que incluye una revisión bibliográfica y contiene un marco temporal de 2013 a 2021. La 
información obtenida se tomó de bases de datos de documentos científicos, seleccionando documentos descritos en inglés y portugués, que informan sobre reacciones con SARS- CoV-2 la interacción de una alimentación saludable en la vida diaria es de hecho muy importante además de otros métodos de cuidado. Las plantas medicinales pueden consolar y combatir diversas enfermedades infecciosas. El organismo, cuando se tiene una infección, es bastante vulnerable ya que los microorganismos dañan provocando deficiencias celulares, incluso si el organismo tiene células que las destruyen, sin embargo necesita nutrientes que hagan que el sistema inmunológico encaje preferentemente, preservando el equilibrio para su funcionamiento Desde la protección del sistema inmunológico El sistema actúa para proteger al cuerpo diariamente de los ataques contra las infecciones, cuando virus como el SARS-CoV-2 dominan el cuerpo, las células toman medidas para combatir el virus y posiblemente obtener una cura o mejora para el individuo. Por lo tanto, con base en la literatura revisada, se concluyó que los adyuvantes como la alimentación saludable y las medicinas a base de hierbas están dispuestos a contribuir al reemplazo de nutrientes capaz de fortalecer la inmunidad del individuo.

Palabras clave: Coronavirus; Alimentos funcionales; Suplementos dietéticos; Plantas medicinales; Inmunidad.

\section{Introdução}

A saúde global está ameaçada por uma pandemia de COVID-19, uma doença respiratória causada pelo novo coronavírus, SARS-CoV-2, que foi identificado pela primeira vez em dezembro de 2019 em Wuhan, China. Em 28 de agosto de 2020, 24,7 milhões de pessoas foram infectadas com o vírus em todo o mundo e aproximadamente 838.000 sucumbiram à doença (Croda, \& Garcia, 2020).

Coronavírus (SARS-CoV-2, COVID-19) apresenta-se como a síndrome respiratória aguda grave, referente à família Coronaviridae, com base em várias pesquisas, o primeiro coronavírus que contaminou os seres humanos, surgiu no ano de 1937 e logo foi isolado, em 1965 de fato apresentou-se como coronavírus, e desde então teve outras variedades de vírus, no ano de 2002 e 2012 teve vários casos de contaminação em vários países, ocasionando a vários indivíduos a óbito (Xia, et al., 2020).

Em dezembro de 2019, o governo da China comunicou a Organização mundial da saúde (OMS) que havia casos de pneumonia desconhecida na cidade de Wuhan, na província de Hubei. Expandindo para vários países entre janeiro e junho de 2020, contaminando milhares de indivíduos, e tornando uma pandemia. Pesquisadores reconheceram que a mutação genética, agredia o organismo se multiplicando, as quais a contaminação através de gotículas respiratórias, o tempo de incubação do vírus 2 a 15 dias, podendo ter complicações, ocasionando o óbito (Croda, \& Garcia, 2020).

A pandemia global causada pela nova doença coronavírus 2019 (COVID-19), para a qual ainda não há vacina ou tratamento eficaz disponível, levou a uma emergência da saúde global. Apesar da falta de dados clínicos, evidências convincentes da literatura sugerem que certos nutracêuticos (como gorduras ômega-3, $\beta$-glucanos, aminoácidos, probióticos, vitaminas e minerais) e compostos à base de plantas derivados de extratos de ervas podem ser usados no auxílio do tratamento dos sintomas de COVID-19 (Montserrat-de la Paz, et al., 2016).

Esses compostos naturais podem não apenas modular a imunidade de uma população suscetível, mas também pode abrir caminho para o desenvolvimento de medicamentos que podem ser usados para tratar COVID-19. Sugere-se, portanto, que a avaliação in vitro e in vivo destes compostos deve ser realizada o mais rápido possível, a fim de contrariar o aumento contínuo do número de casos de COVID-19 (Lee, et al., 2009).

Propostas de pesquisas e testes clínicos sugeriram que alguns tratamentos, incluindo suplementos e fotoquímicos, têm o potencial de ajudar a combater a infecção por coronavírus. Um estudo de Álvares, sugeriu que o risco de infecção pode ser reduzido com a suplementação de vitamina e minerais. O vírus acatar o sistema respiratório por uma resposta inflamatória sistêmica de quadro agudo causado por insultos pulmonares diretos e indiretos (Álvarez, Habtemariam, \& Parra, 2015)

A resposta inflamatória sistêmica com o quadro agudo devido o sistema respiratório não submeter os processos necessários, causado por insultos pulmonares diretos e indiretos. Os sintomas mais comuns no componente respiratório são a tosse seca, com pouca exsudação e a redução, provocando dispneia com aumento do desconforto respiratório. As mudanças no 
padrão funcional pulmonar interferem na gravidade clínica da doença, colocando em risco a função dos músculos respiratórios e intolerância ao exercício físico (Nascimento, et al., 2020).

Alimentação adequada, balanceada, saudável e com níveis adequados de vitaminas e minerais ajudar no fortalecimento do organismo favorecendo uma resposta mais rápida ao invasor. Isso porque alimentos possuem Vitaminas A C e D e minerais tais como o zinco e selênio contribuem para a defesa imunológica, melhorando a quimiotaxia e fagocitose, desempenhando papel importante na função/regulação das respostas imune, celulares e humorais, participando no processo de modulação tanto do sistema imune inato quanto do adaptativo, trazendo resposta antiviral contra infeções respiratórias (Tuma, Granado, Sousa, Lopes, \& Siqueira, 2020).

Segundo Ang, et al. (2020), analisou o potencial dos fitoterápicos tradicionais, que continham um total de 56 ervas, para auxiliar no tratamento de pacientes com COVID-19. As propriedades biológicas das plantas medicinais e dos fitoterápicos estão as de estimular as reações do sistema imune, entre elas, ações imunomoduladoras, conferindo um aumento da resposta imunológica do indivíduo. A ação imunomoduladora que pode aumentar os mecanismos de defesa do hospedeiro é chamada de imunoestimulantes e estimulam os mecanismos que envolvem tanto a imunidade inata quanto a imunidade adquirida, através da ativação de células e mediadores (Braga, \& Silva, 2021).

Subsiste múltiplas alternativas para redução do estresse, através disto providenciará para o sistema imune um melhoramento relativo para enfrentamento do COVID-19. Com relação a inúmeras formas de dispor uma estabilidade no sistema imune, fundamental atribuir diariamente uma alimentação adequada, saudável, balanceada, e os níveis da Vitamina D dentro dos parâmetros. Incluir os alimentos ricos em Vitamina A C e D, apresentará desenvolvimento maior na defesa do organismo, assim beneficiará o sistema imunológico, obtendo atribuições das respostas imune, celulares e humorais, a partir dessas mudanças, responderá ter ações antivirais contra infecções respiratórias (Turma, et al., 2020)

A diversidades de fitoterápicos e plantas medicinais que define uma qualidade das ações de defesas no sistema imunológico, incluindo ações imunomoduladoras, concedendo uma resposta elevada a imunidade dos pacientes. A defesa do sistema imune contribui impulsionando a proteção contra as ações dos hospedeiros, que são denominados de imunoestimulantes, sendo eles inata e adquirida (Braga, \& Silva, 2021).

A associação entre SARS-CoV-2 e o estresse oxidativo como um dos fatores de amplificação do processo inflamatório associado a essa virose parece inequívoco. Infecções virais respiratórias associadas a diferentes processos, dentre eles a produção de citocinas e a inflamação, podem ter como um dos mecanismos desencadeadores o desequilíbrio entre a produção de espécies reativas de oxigênio e o potencial redox, caracterizando um estado de estresse oxidativo crucial para a replicação viral e as manifestações da doença (Dias, Chini, Cardoso, Orrico, \& Pereira, 2020). Desta forma o objetivo do presente estudo, foi realizar um levantamento bibliográfico, em torno dos principais coadjuvantes que podem ser usados para $o$ fortalecimento do sistema imunológico. Além de relacionar o uso do mesmo com o desenvolvimento de anticorpos para combater infecções como as causadas pelo SARS-CoV-2.

\section{Metodologia}

O presente estudo trata-se de uma análise de revisão bibliográfica descritiva explicativa, que incluir estudos sobre alimentos funcionais, fitoterápicos e suplementos que possam auxiliar no fortalecimento do sistema imunológico humano os quais tem relação direta ao combate dos sintomas iniciais do COVID-19.

As informações apresentadas foram obtidas das principais bases de dados de documentos científicos, tais como Scielo, ScienceDirect, scopus, biorxiv. Foram selecionadas publicações nos idiomas inglês e português, as quais apresentavam informações pertinente ao tema da presente revisão. Para realização das buscas nas bases de dados usou-se as seguintes palavras-chave: Alimentos funcionais; Vitaminas funcionais; minerais funcionais; Nutracêuticos; Bioatividade. 


\section{Resultados e Discussão}

\section{Histórico}

No final do ano de 2019, na cidade de Wuhan, província de Hubei, na China, teve início a pandemia pelo vírus SARS - CoV-2, também denominado de COVID-19, e popularmente conhecido como Coronavírus, uma doença infecciosa grave, sendo que até o momento não havia tratamento para combatê-la e nem mesmo vacina para previr a sua propagação (Xia, et al., 2020). Após os primeiros casos, novas contaminações foram identificadas e no dia 11 de março de 2020 a Organização Mundial de Saúde declarou a pandemia, gerou distanciamento social e quarentena no Brasil inteiro (Bomfim, \& Golçalves, 2020).

Esta ação foi necessária porque as formas de contágio envolvem o contato direto ou indireto entre os indivíduos, por meio de gotículas respiratórias, como saliva, tosse e espirros, e, em alguns casos, até mesmo através de urina e fezes dos pacientes contaminadas. Ressalta-se que o tempo de incubação do vírus varia entre 1 a 14 dias (Xia, et al., 2020). Os principais sintomas observados são: febre, fadiga e tosse seca, podendo evoluir para dispneia, chegando até quadros mais complexos, como a Síndrome Respiratória Aguda Grave, que pode levar o indivíduo a óbito (Bomfim, \& Golçalves, 2020).

De uma forma geral, o coronavírus atinge principalmente o sistema respiratório, onde causa pneumonia (Bomfim, \& Golçalves, 2020). Pode causar ainda lesão hepática e cardíaca aguda, insuficiência renal, diarreia, doenças no fígado, e com isso causar infecção generalizada, contribuindo para o óbito implicando envolvimento de múltiplos órgãos (Huang, et al., 2020). Com o avanço dos casos de contaminação vacinas foram sendo desenvolvidas, mas a prevenção ainda é o caminho mais indicado. No tratamento do COVID-19 são realizados exames e ocorre a administração de medicamentos, como os antibióticos e antigripais (Xia, et al., 2020).

A nutrição é uma ciência de grande importância, é estar inserida dentro do contexto de tratamento para patologias agudas e crônicas. E principalmente para aquelas no qual se sabe que não tem um tratamento totalmente estabelecido (Laviano, Koverech, \& Zanetti, 2020).

Várias evidências indicam que muitos suplementos nutricionais de várias especiarias, ervas, frutas, raízes e vegetais podem reduzir o risco ou a gravidade de uma ampla gama de infecções virais, aumentando a resposta imunológica. O uso de compostos naturais pode fornecer suporte profilático e terapêutico alternativo juntamente com a terapia para COVID-19 (Mrityunjaya, et al., 2020).

Para o fortalecimento da imunidade, tornando-se fundamental todas as vitaminas e minerais. Devemos preferir frutas e hortaliças in natura, pois são repletos de antioxidantes que combatem os radicais livres. As carnes também são importantes fontes de zinco. Os lipídios são macromoléculas primordiais para o equilíbrio alimentar, assim cada um contribui de maneira significativa para a manutenção da saúde. Devemos evitar, entretanto, os alimentos industrializados que são extremamente processados (Morais, Maia, Damasceno, Seabra, \& Passos, 2020).

Os fitoterápicos são consumidos mundialmente a muito tempo, de acordo com a Agência Nacional de Vigilância Sanitária (ANVISA), são vegetais ou partes destes que contêm substâncias ou classes de substâncias com ação farmacológica. Portanto a utilização os mesmos são importantes para evitar um possível agravamento do quadro clínico do paciente (BRASIL, 2014).

Ainda que não existam estudos em profundidade sobre a eficácia de alimentos funcionais e fitoterápicos como chás com plantas medicinais, os mesmos podem ser utilizados como coadjuvantes no tratamento da enfermidade (Tuma, et al., 2020). Uma alimentação saudável com nutrientes adequados proporcionar ao organismo fortalecimento ajudando a prevenir possíveis patologias tais como diabetes hipertensão, doenças cardiovasculares e inflamatórias, doenças que podem se agravar com a contaminação por SARS-CoV-2. 
Muitas pessoas são infectadas por SARS-CoV-2 e não apresenta sintomas, os chamados assintomáticos, podendo estar relacionado com o a imunidade inata. Os imunomoduladores são de extrema importância neste contexto pois através dele adquirimos o fortalecimento inicial para combater os sintomas iniciais do COVID-19. Impedindo um possível agravamento da doença, mas até o exato momento não há nada comprovado cientificamente

\section{Alimentos funcionais}

As resoluções $\mathrm{n}^{\circ} 18$ e 19/1999, ANVISA descrevem que a propriedades funcionais dispõe como finalidade metabólica, fisiológica ou nutricionais para o desenvolvimento do organismo humano, ou propriedades saúde estão relacionados com novos alimentação ou constituintes que interagem com à doença quando colocada para à atribuição de cura de doenças não e constatada como alimentos ou constituintes mas sim com produtos que engloba a medicina popular, para isso os alimentos que possui essa finalidade terão que ter relatos científicos e avaliações necessária, e comprovações no registro da Agencia Nacional de Vigilância Sanitária (Brasil, 1999a; Brasil, 1999b) .

A alimento funcional foi estabelecida inicialmente pela primeira vez no Japão em meados de 1980 definido os alimentos que possuir substâncias que auxiliam nas funções metabólicas do organismo que oferecer bem está ao corpo como fundamentais para a saúde. O Japão e o único país até o momento que estabeleceu um processor de aprovação regulamentar específico para os alimentos funcionais, denominados como alimentos específicos para a saúde (Hasler, 1998).

Nas últimas décadas o termo dieta e saúde passou a ser observado com um ponto de vista diferente, os alimentos funcionais são alimentos que possuir nutrientes, que ao serem consumidos além de saciar a fome e nutrir, possuir efeitos terapêuticos e metabólicos sobre o organismo auxiliando na prevenção contra patologias (Hasler, 1998).

A alimentação equilibrada influência o fortalecimento do sistema imunológico, a primeira resposta imune inata do ser humano contra o invasor, têm principal defesa no organismo para a eliminação de vírus e demais patologias, sendo necessário para que tenha efeito contra o antígeno, para não lesar tecidos e órgãos do hospedeiro. A resposta imune inata passar a ser comprometida quando o organismo está com uma alimentação fraco, dando assim espaço para possível invasores microbianos, fúngicos, virais (Silva, Sales, Abreu, \& Chaves, 2020).

Alimentação têm ação no dever do sistema imunológico, controle da importância das infecções. Existem diversas relações entre nutrição, dieta, infecção e imunidade, estão relacionadas (Gasmi, Noor, Tippairote, Dadar, Menzel, \& Bjørklund, 2020).

Alimentação saudável é de extrema importância não somente para prevenção da COVID-19, mas para prevenir de diversas patologias, através dela as células do organismo adquirem macro e micronutrientes essenciais e alguns compostos bioativos são necessários para o apropriado funcionamento do organismo (Gasmi, et al., 2020).

Para garantir a hidratação e não trazer desidratação, e essencial o consumo de alimentos e suplementação adequada com vitaminas e minerais. Parte da abordagem nutricional geral para a prevenção de infecções virais é a suplementação ou fornecimento adequado de vitaminas para reduzir potencialmente o impacto negativo da doença (Barazzoni, et al., 2020).

Sabendo-se que a baixa imunidade pode contribuir para o contágio, especialmente nos casos de gripe e resfriados, se aplica ao contágio por Coronavírus, o que indica que uma alimentação adequada pode fortalecer a imunidade e auxiliar na diminuição de risco de contrair algumas patologias.

Nos casos de inflamação progresso de uma melhor resposta imunológica pode ser incluídos alimentos, plantas que podem estar contribuindo na formulação de produtos diferenciados como em iogurtes, farinhas, cereais, geleias e outros insumos, destinados ao público consumidor que estar em buscas de alimentos saborosos, mas com algumas utilidades vinculadas (Santos, et al., 2020). 
A alimentação, deve ser reforçada o consumo de frutas, legumes e verduras e grãos que possuam efeito antioxidante, como a vitamina $\mathrm{C}$, que é essencial para o organismo e aumenta a imunidade, sendo que sua dose diária pode ser obtida por meio do consumo limão, laranja, acerola etc. Alimentos que contenham ferro também devem ser consumidos, porque a deficiência desta vitamina gera anemia e baixa a imunidade, aumentando as chances de contágio. O selênio é outra vitamina antioxidante e que pode ser obtida por meio do consumo de 2 unidades de castanha do Pará (Tuma, et al., 2020).

Independentemente de serem insuficientes, existem alimentos em que possui concentrações satisfatórias de vitamina C, para ingestão oral, como óleo de fígado de peixe, carnes e frutos do mar e alguns outros tipos, incluindo os alimentos fortificados, geralmente utilizados para prevenção de quadros clínicos, ligados a hipovitaminose D (Dutra, et al., 2020).

A suplementação com vitaminas e minerais como vitamina A, B, C, D, ferro e zinco também são bem-aceitas, de acordo com as necessidades diárias de cada indivíduo (Lima, 2020). Além da alimentação a boa hidratação é de extrema relevância no COVID-19. De acordo com Morais os vírus podem causar redução do apetite e causar desidratação, diarreia e vômito (Morais, et. al., 2020).

A alimentação obtém compostos bioativos o consumo de nutrientes é importante, entretanto são antioxidantes, antiinflamatórios e imunomoduladores do sistema imunológicos. Os alimentos ricos em vitamina $\mathrm{C}$, vitamina $\mathrm{D}$, zinco, selênio, Ferro dentre outros, são nutrientes que auxiliar na modulação das células da função imunológicas (Cheng, 2020). Portanto o estado nutricional de um indivíduo pode determinar o risco de agravamento da infecção por SARS-CoV-2. A manutenção de uma alimentação saudável ricas em micronutriente pode ser uma medida de prevenção ao novo coronavírus.

\section{Vitamina C}

$\mathrm{O}$ ácido ascórbico conhecido popularmente como vitamina $\mathrm{C}$ é um importante nutriente para organismo humano, porém ele não é capaz de produzi-lo. Assim como também é incapaz de armazená-lo em grandes quantidades por ser hidrossolúvel e termolábil, tornando necessário o consumo regular de alimentos que contenha este nutriente. Com a deficiência do ácido ascórbico ocorre o desenvolvimento de escorbuto doença que enfraquece as estruturas de colágenos, ocasionando uma má cicatrização de ferimentos, prejudicando a imunidade. Pacientes com carência de vitamina $\mathrm{C}$ são suscetivelmente propicio a adquirir infecções fatais (Carr, \& Maggini, 2017).

A vitamina $\mathrm{C}$ e um imunomodulador importante, ela contribui para a defesa do sistema imunológico. Possuir funções em diversas células do sistema imunitário inato e adaptativa, amplificando assim sua capacidade de proteção contra infecções. Sua ação antioxidante e altamente eficaz devido sua habilidade de doar elétrons protegendo biomoléculas importantes. Sendo excelente na eliminação dos oxidantes na pele, protegendo contra o estresse oxidativo, no caso do neutrófilos pode auxiliar na inibição da oxidação, pois o mesmo possui alto nível de ácido graxo, no entanto quando a vitamina $\mathrm{C}$ entra em contato com a membrana onde está acoplado o ácido graxo, reduz a oxidação, sendo assim reestrutura a vitamina E (Carr, et al., 2017).

Os níveis de vitamina $\mathrm{C}$ no sangue diminuir no período de infecção, sendo necessário a administração para restaurara os níveis normais (Lotfi, Hamblin, \& Rezaei, 2020). Sua fonte de nutrição são principalmente frutas cítricas, verduras e algumas especiarias. Os glóbulos brancos contêm mais vitamina C, que o plasma, demostrando funções funcionais nas células do sistema imunológica, essencial para o organismo aumentando imunidade (Mrityunjaya, et al., 2020).

A ação da vitamina $\mathrm{C}$ em vitro ou vivo com concentrações adequada de dose em respectivos casos, apresenta efeitos sendo eles vasodilatação e antioxidantes (Padayatty, et al., 2016).

De acordo com Biancatelli (2020), relata que vitamina C associada com outros medicamentos traz um desempenho para auxiliar em tratamentos infecciosos virais, em doses altas possui neutralização ou até mesmo destrói o vírus, tendo em vista que a vitamina $\mathrm{C}$ é um antioxidante sendo assim as células será protegida contra ações que possa provocar lesões. A vitamina $\mathrm{C}$ tem propriedade imunomoduladoras poderá tendo os amplos benefícios, o que se pode observar é que pessoas que 
estão com quadros infecioso tem pouca vitamina $\mathrm{C}$ no organismo, através da análise clínicas desenvolvida obtivemos informações cuja o papel da vitamina $\mathrm{C}$ contribui para os sistemas imunes, quando o corpo infectado pelo vírus da gripe e herpes.

\section{Vitamina D}

A vitamina D são dois esteroides vitamina D2 e vitamina D3, ambas são obtidas através da alimentação, mas a vitamina D3 ela e sintetizada através da exposição a luz solar, principal fonte de D3 (Colecalciferol). A vitamina D e extremamente fundamental para impulsionar o sistema imunológico e reduz as gravidades das infecções do trato respiratório (Kechichian, et al., 2018), A vitamina D extremamente relevante no sistema imunológico, devido ao seu poder de retirar vírus envelopados e realizar a liberação de macrófagos e diminuir a produção de citocinas pró-inflamatórias, que é provocada pela fisiopatologia da SARS-CoV-2 (Grant, Baggerly, \& Lahore, 2020).

De acordo com Rodrigues (2020) SARS-CoV-2 lesa células epiteliais do pulmão e promover o desenvolvimento de pneumonia por produzir níveis elevados de citocinas pro-inflamatórias, auxiliar na diminuição da cascata de citocinas. A falta de vitamina D inibir a capacidade dos macrófagos em evitar liberação das citocinas pro-inflamatórias (Rodrigues, et al., 2020). Portanto e essencial a manutenção de vitamina D no organismo, embora a fonte principal de seja a exposição a luz solar, existe alguns alimentos que auxiliar a obtenção, como gema de ovo, salmão, sardinha e outros alimentos (Lichtenstein, et al., 2013).

A vitamina $\mathrm{D}$, obtida a partir da exposição da pele a luz solar, também produz efeitos positivos no fortalecimento da imunidade e reduz quadros depressivos que possam agravar a enfermidade (Tuma, et al., 2020).

Deficiência de vitamina D ocorre sintomas de fraqueza e dores, portanto, paciente que se encontra em tratamento em UTI, necessita associada com outra medicação, visto que para o sistema imunológicos seja indispensável, para não afetar em casos de infecção por virucidas. Em casos de insuficiência possivelmente ter contrações de tuberculose, infecções respiratórias, doenças transmissíveis. Estudo em paciente com coronavírus, que possui deficiência de vitamina D o quadro de mortalidade e maior, a pesquisa conforme desenvolvida através Yisak (2021) demostrou que os indivíduos com ausências de vitamina D há gravidade.

Vitamina D fator importante, possui dois tipos de manifestar de forma vegetais que são conhecidas com D2 ergocalciferol que vem de plantas, fungos e exposição ao sol, a D3 colecalciferol são produzidas pela exposição ao sol onde se implanta na pele e em alimentos. Considere-se que a falta de vitamina D proporciona várias doenças, como câncer, insuficiência na imunidade inata e adquirida, desenvolver inflamações. Portanto a vitamina D influencia em variadas doenças sendo elas repiratorias, infeciosas e patógenos. De acordo com esse estudo pode ser uma alternativa para o tratamento do Coronavírus (Silvino, et al., 2020)

\section{Zinco (Zn)}

O zinco tem micronutriente essencial, possuir ação antioxidante, possuir um papel no desempenho do sistema imunológico tanto inato quanto adaptativo auxiliar no desenvolvimento e manutenção das células, atuar na regulamentação diferenciação e proliferação e funcionamento de linfócitos e leucócitos (Maares, \& Haase 2016). Segundo Gammoh (2017) baixo nível no organismo, pode apresentar alterações no sistema imune, aumentado portando o potencial a suscetibilidade a patologias inflamatórias e infecções (Gammoh, \& Rink, 2017)

O zinco aumenta as atividades citotóxicas das células Natural Killer (NK) são celular importante na vigilância da imunidade inata, as NK atacar e matam as células anormais na membrana plasmática, tem ação anti-inflamatória pois ele está envolvido na produção de anticorpos (Daltoe, \& Demoliner, 2020). Sendo assim a deficiência de Zn provocar o aumento da suscetibilidade a infecções, causadas por microrganismo como bacterianos, fúngicos e virais (Panyod, Ho, \& Sheen, 2020). 
A dose terapêutica apropriado suplemento de Zn possui um potencial de melhor a função do sistema imune, fornecendo ação antiviral de maneira sinérgica na terapia padrão. As principais fontes de Zinco são leite castanha de caju, amendoim e ovos. (Panyod, Ho, \& Sheen, 2020). Portanto o zinco tem ação na ativação imunológica, diminuído assim a probabilidade de infecções. Os alimentos que contenham ferro também devem ser consumidos, porque a deficiência desta gera anemia e baixa a imunidade, aumentando as chances de contágio.

$\mathrm{O}$ zinco apontado como excelente em funções estruturais de proteínas, considerando que participa de enzimas antioxidantes, a interação do zinco com o sistema imune apresenta ter amplos benefícios nas células $\mathrm{T}$, que proporciona a produção de outras células para a defesa do corpo. Alguns casos clínicos declaram que incapacitação do zinco no organismo correlaciona com desnutrição, tendo insuficiência na formação anticorpos, nesse caso clinico ocorreu in vivo no qual os camundongos, dispôs do tratamento realizado na medula óssea, constatando que a deficiência do zinco, capaz de modificar as células vermelhas. Desfecho do caso clínico portam de leves a graves sintomas que a infecção acomete, assim sendo os indícios de dermatites, anorexia, complicações emocionais, etc. mediante incapacidade do organismo de restabelecer, propõem dieta para o paciente para repor a nutrição necessária, com suplementos, alimentos que preserve o zinco no organismo (Sarni, Souza, Cocco, Mallozi, \& Solé, 2010).

\section{Selênio}

O selênio é outro nutriente que auxiliar no antioxidante e que pode ser obtida por meio do consumo de 2 unidades de castanha do Pará. Muitas pessoas são infectadas por SARS-CoV-2 e não apresenta sintomas os chamados assintomáticos, podendo estar relacionado com o a imunidade. Os imunomoduladores são de extrema valor neste contexto através dele adquire-se o fortalecimento inicial para auxiliar no combater os sintomas iniciais do COVID-19, impedindo um possível agravamento da doença (Tuma, et al., 2020). Não a estudos que comprove que tem alguma substância que impeça a infecção por SARS-CoV-2, porém sabe-se que um organismo com uma boa nutrição previne possíveis patologias.

\section{Fitoterápicos}

As plantas dispõem de propriedades medicinais das formas mais antigas da prática medicinal, sendo utilizada para o tratamento, cura e prevenção de doenças (Quresh, et al., 2016). Esta aplicação antiga ocorre em razão aos estudos a longo prazo para obtenção de recursos naturais alternativos para melhoria da qualidade de vida. As plantas medicinais funcionam como matéria-prima para drogas, fitoterápicos ou como agentes terapêuticos isolados, em chás, macerados e cozimento (Brasil, 2010)

A população optou por plantas medicinais como método caseiro destinado a produção de remédios naturais, dessa forma o processo é preparado pelo próprio indivíduo em sua residência. Estudos apontam que $80 \%$ da população mundial faz uso de fitoterápicos a procura do conforto para dores, a utilização é de fácil acesso, baixo custo e por serem consideradas inofensivas por grande parte da população (Zeni, Parisotto, Mattos, \& Helena, 2017).

As plantas medicinais e os fitossociologias possuir atributos biológicos que são capazes ativar as ações estimulantes das reações do sistema imunológicas, induzindo assim as atividades imunomoduladoras das células do hospedeiro, isso acontece, pois, a ativação das células mediadoras que fortalece tanto a resposta inata quanto adaptativa (Nunes-Pinheiro, Leite, Farias, Braga, \& Lopes, 2003).

Conforme o artigo de Khan algumas plantas medicinais, que são utilizadas para o tratamento de doenças respiratórias causada por vírus, como a Forsythia, e várias outras controla e alivia os sintomas como a ação anti-inflamatória, broncodilatadora, expectorante, analgésica e antipirética, matéria-prima para medicamentos antivirais (Khan, et. al., 2020) 
No enfrentamento da SARS-CoV-2, diversos indivíduos estão recorrendo ao método tradicional associado ao uso de plantas medicinais. Assim as plantas medicinais têm colaborado para a prevenção, há estudos mencionam a sua capacidade (Thuy, et al., 2020).

A fitoterapia é um método de tratamento caracterizado pela utilização de plantas medicinais e fitoterápicos em suas diversas preparações, constituindo uma modalidade de terapia integrativa e complementar diante das necessidades de saúde e seu uso tem sido crescente na população de diversos países (Figueredo, Gurgel, \& Gurgel-Junior, 2014).

As partes das plantas mais utilizadas são principalmente as folhas e a forma de preparo mais utilizada nas comunidades é o chá, por infusão, sendo que a maceração, banhos, inalação, pomada e cataplasma são as demais formas de uso também encontradas (Oliveira, \& Menini Neto, 2012).

A presença de compostos fenólicos, carotenoides, antocianinas e flavonoides nas flores da espécie Viola tricolor, conhecida popularmente como amor perfeito, sua elevada taxa de atividade antioxidante o que representa grande importância nos processos inflamatórios de várias doenças como as cardiovasculares, câncer, dentre outras patologias, sendo ainda, um potente estimulador do sistema imunológico, por ocasionar diminuição do estresse oxidativo (Silva, et al., 2020).

Outra forma de auxiliar no fortalecimento do sistema imunológico dos indivíduos é por meio de plantas medicinais, como chás e infusões, porque os produtos naturais podem, até mesmo, servirem como base para o desenvolvimento de vacinas e medicações que cooperem com o enfrentamento do COVID-19 (Silva, et al., 2020).

Diversos artigos científicos têm estudado e apontam que as plantas medicinais são de extrema importância. São opção a vários medicamentos sintéticos. Essas plantas apresentam funcionalidades in vitro, e in vivo. Esses preparados funcionais são produzidos a partir de fontes naturais, incialmente disseminado por meio de conhecimentos de nativos que já fazem o uso (Silva, et al., 2020; Santos et al., 2020)

Destaca-se ainda a importância de recursos terapêuticos típicos da Aromaterapia e Fitoterapia, tais como: infusões, preparos caseiros com plantas aromáticas, como chás de ervas e plantas medicinais. Estes podem aliviar os sintomas físicos e emocionais decorrentes do COVID-19 (Nascimento \& Prade, 2020).

O uso de Eucalipto para inalação é amplamente indicado para o alivio dos sintomas de resfriados e infecções das vias respiratórias, sendo considerado seguro e eficaz. A menta japonesa, também conhecida como vick ou hortelã, é reconhecida pelo alto teor de mentol o que faz com que seja indicada para a mesma finalidade. Ressalta-se que sua administração dispensa o uso de chás, bastando apenas esmagar algumas folhas e inalar o cheiro. Já a erva cidreira possui propriedades antivirais e expectorantes, sendo popularmente utilizada para desobstruir as narinas (Pereira, et al., 2017).

Plantas medicinais que tem na sua composição substâncias que desenvolve atividade imunomoduladora, sendo elas polissacarídeos, lectinas, peptídeos, saponinas, óleos e outras, dando impulso para o sistema imune, são utilizadas pela população indígenas (Nunes-Pinheiro, et al., 2003).

Portanto os resultados apresentados após a pesquisa demostrar que os fitoterápicos e a aromaterapia tem ação imunomodulador sobre o sistema imunológico auxiliando, portanto, no alívio de sintomas do COVID-19, podendo ser promissor a descoberta de novos medicamentos, neste contexto espera-se que seja desenvolvido mais pesquisas na tentativa de descobrir novos tratamentos.

\section{Considerações Finais}

A pesquisa apresentada mostrou por meio de artigos científicos, substâncias que atuam no sistema imunológico. Desta forma proporcionando benefícios ao funcionamento do corpo, promovendo o fortalecimento do sistema imune, para as células se autodefender contra vírus e bactérias que são capazes de trazer danos ao organismo. Com relação as plantas medicinais, elas apresentam uma rica composição química (metabolitos secundários) a qual faz com que desempenhe funcionalidades benéficas 
a saúde humana. É valido salientar que, há uma grande variabilidade de funcionalidade, o que depende diretamente da composição química específica de cada vegetal.

É importante ressaltar que a alimentação equilibrada, substâncias bioativas, exercício físico e o consumo regular de água, contribuirá para que o mecanismo de defesa do corpo humano apresente melhor desempenho. Assim com o consumo exagerado de sal, açúcares e gorduras não é apropriado para essa função de fortalecimento do sistema imunológico.

\section{Referências}

Álvarez, Á. L., Habtemariam, S., \& Parra, F. (2015). Inhibitory effects of lupene-derived pentacyclic triterpenoids from Bursera simaruba on HSV-1 and HSV-2 in vitro replication. Natural Product Research, 29(24), 2322-2327. https://doi.org/10.1080/14786419.2015.1007456

Ang, L., Lee, H. W., Kim, A., Lee, J. A., Zhang, J., \& Lee, M. S. (2020). Herbal medicine for treatment of children diagnosed with COVID-19: A review of guidelines. Complementary therapies in clinical practice, 39, 101174. https://doi.org/10.1016/j.ctcp.2020.101174.

Barazzoni, R., Bischoff, S. C., Breda, J., Wickramasinghe, K., Krznaric, Z., Nitzan, D., Pirlich, M., \& Singer, P. (2020). ESPEN expert statements and practical guidance for nutritional management of individuals with SARS-CoV-2 infection. Liječnički vjesnik, 142(3-4), 75-84.

Braga, J. C. B., \& Silva, L. R. (2021). Consumo de plantas medicinais e fitoterápicos no Brasil: perfil de consumidores e sua relação com a pandemia de COVID-19. Brazilian Journal of Health Review, 4(1). https://doi.org/10.34119/bjhrv4n1-303.

Biancatelli, R. M. L. C., Berrill, M., \& Marik, P. E. The antiviral properties of vitamina C, Expert Review of Anti-infective Therapy, 18: 2, 99-101, 2020. https://doi.org/10.1080/14787210.2020.1706483.

Brasil. Agência Nacional de Vigilância Sanitária - ANVISA. RDC Nº10, DE 09 DE MARÇO DE 2010. Dispõe sobre a notificação de drogas vegetais junto à Agência Nacional de Vigilância Sanitária (ANVISA) e dá outras providências. Diário Oficial da União, Brasília.

Brasil. Agência Nacional de Vigilância Sanitária- ANVISA. RCD Nº18, DE 30 DE ABRIL DE 1999. Aprova o Regulamento Técnico de procedimentos para registro de alimento com alegação de propriedades funcionais e ou de saúde em sua rotulagem. Diário Oficial da União, Brasília.

Brasil. Agência Nacional de Vigilância Sanitária- ANVISA. RCD N¹9, DE 19 DE NOVEMBRO DE 1999. Aprova o Regulamento Técnico de procedimentos para registro de alimento com alegação de propriedades funcionais e ou de saúde em sua rotulagem. Diário oficial da união, Brasília.

Brasil. Agência Nacional de Vigilância Sanitária- ANVISA. RDC Nº 26 DE 13 DE MAIO DE 2014. Dispõe sobre o registro de medicamentos fitoterápicos e o registro e a notificação de produtos tradicionais fitoterápicos. Diário oficial da união, Brasília.

Bomfim, J. H. G. G., \& da Silveira Gonçalves, J. (2020). Suplementos alimentares, imunidade e COVID-19: qual a evidência? VITTALLE-Revista de Ciências da Saúde, 32(1), 10-21. https://doi.org/10.14295/vittalle.v32i1.11282.

Carr, A. C., \& Maggini, S. (2017). Vitamin C and immune function. Nutrients, 9(11), 1211. https://doi.org/10.3390/nu9111211.

Chan, J. F. W., Yip, C. C., To, K. K., Tang, T. H., Wong, S. C., Leung, K., Fung, A. Y., Ng, A. C., Zou, Z., Tsoi, H., Choi, G. K., Tam, A. R., Cheng, V. C., Chan, K., Tsang, O. T., \& Yuen, K. Y. (2020). Improved molecular diagnosis of COVID-19 by the novel, highly sensitive and specific COVID-19-RdRp/Hel real-time reverse transcription-PCR assay validated in vitro and with clinical specimens. Journal of clinical microbiology, 58(5), e00310-20. https://doi.org/10.1128/JCM.00310-20.

Cheng, Z. J., \& Shan, J. (2020). 2019 Novel coronavirus: where we are and what we know. Infection, 48(2), 155-163. https://doi.org/10.1007/s15010-020$01401-\mathrm{y}$.

Croda, J. H. R., \& Garcia, L. P. (2020). Resposta imediata da Vigilância em Saúde à epidemia da COVID-19. https://doi.org/10.5123/S167949742020000100021.

Daltoe, L. M., \& Demoliner, F. (2020). COVID-19: nutrição e comportamento alimentar no contexto da pandemia. Revista Perspectiva: Ciência e Saúde, 5(2).

Dias, M. J. L. E., Chini, M. C. Cardoso, T. F., Orrico, S. R. P., \& Pereira, B. L. B. (2020). COVID-19 e Nutrição. Ulakes Journal of Medicine, 1.

Dutra, J. M., Brito, I. S., Maia, G. P. A. G., Faria, A. B., Chagas, P. P., \& Plácido, G. R. (2020). Deficiência e biodisponibilidade da vitamina D: Uma revisão bibliográfica. Research, Society and Development, 9(7), e23973555-e23973555. https://doi.org/10.33448/rsd-v9i7.3555.

Figueredo, C. A. D., Gurgel, I. G. D., \& Gurgel Junior, G. D. (2014). A Política Nacional de Plantas Medicinais e Fitoterápicos: construção, perspectivas e desafios. Physis: Revista de Saúde Coletiva, 24, 381-400. https://doi.org/10.1590/S0103-73312014000200004.

Gasmi, A., Noor, S., Tippairote, T., Dadar, M., Menzel, A., \& Bjørklund, G. (2020). Individual risk management strategy and potential therapeutic options for the COVID-19 pandemic. Clinical Immunology, 215, 108409. https://doi.org/10.1016/j.clim.2020.108409.

Gammoh, N. Z., \& Rink, L. (2017). Zinc in infection and inflammation. Nutrients, 9(6), 624. https://doi.org/10.3390/nu9060624.

Grant, W. B., Baggerly, C. A., \& Lahore, H. (2020). Reply:"Vitamin D supplementation in influenza and COVID-19 infections. Comment on: Evidence that vitamin D supplementation could reduce risk of influenza and COVID-19 infections and deaths nutrients 2020 , 12 (4), 988". Nutrients, 12(6), 1620. https://doi.org/10.3390/nu12061620.

Hasler, C. M. (1998). Functional foods: their role in disease prevention and health promotion. Food Technology-Champaign Then Chicago-, 52, 63-147. 
Huang, C., Wang, Y., Li, X., Ren, L., Zhao, J., Hu, Y., Xia J., Wei Y., Wu W., Xie X., Yin W., Li H., Liu M., Xiao Y., Gao H., Guo L., Xie J., Wang G., Jiang R., Gao Z., Jin Q., Wang J., \& Cao, B. (2020). Clinical features of patients infected with 2019 novel coronavirus in Wuhan, China. The lancet, 395(10223), 497-506. https://doi.org/10.1016/S0140-6736(20)30183-5.

Kechichian, E., \& Ezzedine, K. (2018). Vitamin D and the skin: an update for dermatologists. American journal of clinical dermatology, 19(2), 223-235. https://doi.org/10.1007/s40257-017-0323-8.

Khan, S., Siddique, R., Shereen, M., Ali, A., Liu, J., Bai, Q., Bashir, N., \& Xue,M. (2020). Emergence of a novel coronavirus, severe acute respiratory syndrome coronavirus 2: biology and therapeutic options. Journal of clinical microbiology, 58(5), e00187-20. https://doi.org/10.1128/JCM.00187-20 .

Laviano, A., Koverech, A., \& Zanetti, M. (2020). Nutrition support in the time of SARS-CoV-2 (COVID-19). Nutrition (Burbank, Los Angeles County, Calif.), 74, 110834. https://doi.org/10.1016/j.nut.2020.110834.

Lee, C., Lee, J. M., Lee, N. R., Kim, D. E., Jeong, Y. J., \& Chong, Y. (2009). Investigation of the pharmacophore space of Severe Acute Respiratory Syndrome coronavirus (SARS-CoV) NTPase/helicase by dihydroxychromone derivatives. Bioorganic \& medicinal chemistry letters, 19(16), 4538-4541. https://doi.org/10.1016/j.bmcl.2009.07.009.

Lichtenstein, A., Ferreira-Júnior, M., Sales, M. M., Aguiar, F. B. D., Fonseca, L. A. M., Sumita, N. M., \& Duarte, A. J. (2013). Vitamina D: ações extraósseas e uso racional. Revista da Associação Médica Brasileira, 59, 495-506. https://doi.org/10.1016/j.ramb.2013.05.002.

Lima, C. M. A. D. O. (2020). Informações sobre o novo coronavírus (COVID-19). https://doi.org/10.1590/0100-3984.2020.53.2e1.

Lotfi, M., Hamblin, M. R., \& Rezaei, N. (2020). COVID-19: Transmission, prevention, and potential therapeutic opportunities. Clinica chimica acta, 508, 254-266.

Maares, M., \& Haase, H. (2016). Zinc and immunity: An essential interrelation. Archives of biochemistry and biophysics, 611, 58-65. https://doi.org/10.1016/j.abb.2016.03.022.

Morais, A. H. A., Maia, J. K. S., Damasceno, K. S. F. S. C., Seabra, L. M. J., \& Passos, T. S. (2020). Orientações nutricionais para o enfrentamento do COVID-19. Universidade Federal do Rio Grande do Norte, 2020.

Mrityunjaya, M., Pavithra, V., Neelam, R., Janhavi, P., Halami, P. M., \& Ravindra, P. V. (2020). Immune-boosting, antioxidant and anti-inflammatory food supplements targeting pathogenesis of COVID-19. Frontiers in Immunology, 11. https://doi.org/10.3389/fimmu.2020.570122.

Montserrat-de la Paz, S., Fernandez-Arche, A., De La Puerta, R., Quilez, A. M., Muriana, F. J., Garcia-Gimenez, M. D., \& Bermudez, B. (2016). Mitraphylline inhibits lipopolysaccharide-mediated activation of primary human neutrophils. Phytomedicine, 23(2), 141-148. https://doi.org/10.1016/j.phymed.2015.12.015.

Nascimento, J. H. P., Gomes, B. F. D. O., Carmo, P. R. D., Petriz, J. L. F., Rizk, S. I., Costa, I. B. S. D. S., Lacerda, M. V. G., Bacal, F., Hajjar, L. A., \& Oliveira, G. M. M. D. (2020). COVID-19 e estado de hipercoagulabilidade: uma nova perspectiva terapêutica. Arquivos Brasileiros de Cardiologia, 114, 829833.

Nascimento, A., \& Prade, A. C. K. (2020). Aromaterapia: o poder das plantas e dos óleos essenciais. Recife: Fiocruz-PE.

Nunes-Pinheiro, D. C. S., Leite, A. K. R. M., Farias, V. M., Braga, L. T., \& Lopes, C. A. P. Atividade imunomoduladora das plantas medicinais: perspectivas em medicina veterinária. Ciência Animal, 13(1):23-32, 2003.

Padayatty, S. J., \& Levine, M. (2016). Vitamin C: the known and the unknown and Goldilocks. Oral diseases, 22(6), 463-493. https://doi.org/10.1111/odi.12446.

Panyod, S., Ho, C. T., \& Sheen, L. Y. (2020). Dietary therapy and herbal medicine for COVID-19 prevention: A review and perspective. Journal of traditional and complementary medicine, 10(4), 420-427. https://doi.org/10.1016/j.jtcme.2020.05.004.

Oliveira, E. R., \& Menini Neto, L. (2012). Levantamento etnobotânico de plantas medicinais utilizadas pelos moradores do povoado de Manejo, Lima DuarteMG. Revista Brasileira de plantas medicinais, 14, 311-320.

Pereira, A. M. S. (2017). Formulário de Preparação Extemporânea - Farmácia da Natureza - chás medicinais. São Paulo: Bertolucci.

Qureshi, R., Ghazanfar, S. A., Obied, H., Vasileva, V., \& Tariq, M. A. (2016). Ethnobotany: a living science for alleviating human suffering. https://doi.org/10.1155/2016/9641692.

Rodrigues, T. S., Sá, K. S. G., Ishimoto, A. Y., Becerra, A., Oliveira, S., Almeida, L., Gonçalves, A. V., Perucello, D. B., Andrade, W. A., Castro, R., VERAS, F. P., Toller-Kawahisa, J. E., Nascimento, D. C., Lima, M. H. F., Silva, C. M. S., Caetite, D. B., Martins, R. B., Castro, I. A., Pontelli, M. C., Barros, F. C., Amaral, N. B., Giannini, M. C., Bonjorno, L. P., Lopes, M. I. F., Santana, R. C., Vilar, F. C., Auxiliadora-Martins, M., Luppino-Assad, R., Almeida, S. C. L., Oliveira, F. R., Batah, S. S., Siyuan, L., Benatti, M. N., Cunha, T. M., Alves-Filho, J. C., Cunha, F. Q., Cunha, L. D., Frantz, F. G., Kohlsdorf, T., Fabro, A. T., Arruda, E., Oliveira, R. D. R., Louzada-Junior, P., \& Zamboni, D. S. (2020). Inflammasomes are activated in response to SARS-CoV-2 infection and are associated with COVID-19 severity in patients. Journal of Experimental Medicine, 218(3), e20201707. https://doi.org/10.1084/jem.20201707.

Silva, L. H. S., Sales, I. O. M., Abreu, D. C., \& Chaves. A. (2020). O Papel da Imunidade Inata na COVID-19. Health Sciences Journal, 10(3), 5-8.

Santos, J. S., Escher, G. B., Carmo, M. V., Azevedo, L., Marques, M. B., Daguer, H., Molognoni, L., Genovese, M. I., Wen, M., Zhang, L., Oh, G. J., Shahidi, F., \& Granato, D. (2020). A new analytical concept based on chemistry and toxicology for herbal extracts analysis: From phenolic composition to bioactivity. Food Research International, 132, 109090. https://doi.org/10.1016/j.foodres.2020.109090

Sarni, R. O., Souza, F. I., Cocco, R. R., Mallozi, M. C., \& Solé, D. (2010). Micronutrientes e sistema imunológico. Rev Bras Alerg Imunopatol, $33(1), 8$-13. 
Research, Society and Development, v. 10, n. 13, e111101320983, 2021

(CC BY 4.0) | ISSN 2525-3409 | DOI: http://dx.doi.org/10.33448/rsd-v10i13.20983

Silva, F. G. C., Borges, A. L. T. F., de Oliveira, J. V. L., do Nascimento Prata, A. P., de Moraes Porto, I. C. C., de Almeida, C. A. C., Sousa, J.S., Freitas, J.D., Oliveria-Filho, A. D., Reis, F.M.P. Oliveira, R. A. G., Silva, S.A.S., \& Nascimento, T. G. (2020). Alimentos, Nutracêuticos e Plantas Medicinais Utilizados como Prática Complementar no Enfrentamento dos Sintomas do Coronavírus (Covid-19): Uma Revisão. https://doi.org/10.1590/SciELOPreprints.317.

Silvino, V. O., Pereira, M. M. L., de Moura, R. C., Batista, M. C. C., Rosa, B. V., de Moura, E. H., ... \& dos Santos, M. A. P. (2020). Vitamina D e doenças infectocontagiosas na pandemia da COVID-19. Research, Society and Development, 9(7), e771974614-e771974614. https://doi.org/10.33448/rsd-v9i7.4614.

Tuma, R., Granado, T., Sousa, G., Lopes, D., \& Siqueira, B. (2020). Alimentação e Nutrição em tempos de pandemia - Esclareçam as suas dúvidas. "Pará contra o Coronavírus". Secretaria de Saúde Pública, Governo do PARÁ.

Thuy, B. T. P., My, T. T. A., Hai, N. T. T., Hieu, L. T., Hoa, T. T., Thi Phuong Loan, H., Triet, N. T., Anh, T. T. V., Quy, P. T., Tat, P. V., Hue, N. V., Quang, D. T., Trung, N. T., Tung, V. T., Huynh, L. K., \& Nhung, N. T. A. (2020). Investigation into SARS-CoV-2 resistance of compounds in garlic essential oil. ACS omega, 5(14), 8312-8320. https://doi.org/10.1021/acsomega.0c00772.

Xia, S., Lan, Q., Su, S., Wang, X., Xu, W., Liu, Z., Zhu, Y., Wang, Q., Lu, L., \& JIANG, S. (2020). The role of furin cleavage site in SARS-CoV-2 spike protein-mediated membrane fusion in the presence or absence of trypsin. Signal transduction and targeted therapy, 5(1), 1-3. https://doi.org/10.1038/s41392020-0184-0.

Xia, S., Lan, Q., Su, S., Wang, X., Xu, W., Liu, Z., Zhu, Y., Wang, Q., Lu, L., \& Jiang, S. (2020). Inhibition of SARS-CoV-2 (previously 2019-nCoV) infection by a highly potent pan-coronavirus fusion inhibitor targeting its spike protein that harbors a high capacity to mediate membrane fusion. Cell research, 30(4), 343-355. https://doi.org/10.1038/s41422-020-0305-x.

Yisak, H., Ewunetei, A., Kefale, B., Mamuye, M., Teshome, F., Ambaw, B., \& Yitbarek, G. Y. (2021). Effects of vitamin D on COVID-19 infection and prognosis: a systematic review. Risk management and healthcare policy, 14, 31. https://doi.org/10.2147/RMHP.S291584.

Zeni, A. L. B., Parisotto, A. V., Mattos, G., \& Helena, E. T. D. S. (2017). Utilização de plantas medicinais como remédio caseiro na Atenção Primária em Blumenau, Santa Catarina, Brasil. Ciência \& Saúde Coletiva, 22, 2703-2712. https://doi.org/10.1590/1413-81232017228.18892015. 